\begin{tabular}{|c|c|}
\hline Title & $\begin{array}{l}\text { Brain-derived neurotrophic factor induces angiogenin secretion and nuclear translocation in human umbilical vein } \\
\text { endothelial cells }\end{array}$ \\
\hline Author(s) & $\begin{array}{l}\text { Mori, A yako; Nishioka, Y usuke; Y amada, Mai; Nishibata, Y uka; Masuda, Sakiko; Tomaru, Utano; Honma, Naoyuki; } \\
\text { Moriyama, Takanori; Ishizu, A kihiro }\end{array}$ \\
\hline Citation & $\begin{array}{l}\text { Pathology - Research and Practice, 214(4), 521-526 } \\
\text { https://doi.org/10.1016/.prp.2018.02.013 }\end{array}$ \\
\hline Issue Date & 201804 \\
\hline Doc URL & http:/hdl.handle.net/2115/73833 \\
\hline Rights & $\begin{array}{l}\text { () 2018. This manuscript version is made available under the CC-BY-NC-ND } 4.0 \text { license } \\
\text { http://creativecommons.org/icenses/by-nc-nd/4.0/ }\end{array}$ \\
\hline Rights(URL) & http://creativecommons.org/icenses/by-nc-nd/4.0/ \\
\hline Type & article (author version) \\
\hline File Information & Pathology Research and Practice_2018.pdf \\
\hline
\end{tabular}

Instructions for use 


\section{Brain-derived neurotrophic factor induces angiogenin secretion and nuclear translocation in human umbilical vein endothelial cells}

\section{Running Title: BDNF and angiogenin}

Ayako Mori ${ }^{1}$, Yusuke Nishioka ${ }^{1}$, Mai Yamada ${ }^{1}$, Yuka Nishibata $^{2}$, Sakiko Masuda $^{2}$, Utano Tomaru ${ }^{3}$, Naoyuki Honma ${ }^{2}$, Takanori Moriyama ${ }^{2}$, Akihiro Ishizu ${ }^{2, *}$

${ }^{1}$ Graduate School of Health Sciences, Hokkaido University, Sapporo, Japan

${ }^{2}$ Faculty of Health Sciences, Hokkaido University, Sapporo, Japan

${ }^{3}$ Department of Pathology, Faculty of Medicine and Graduate School of Medicine, Hokkaido University, Sapporo, Japan

*Corresponding author: Akihiro Ishizu, MD, PhD

Faculty of Health Sciences, Hokkaido University, Sapporo 0600812, Japan

Tel. +81 11706 3385; fax: +81 117064916

E-mail: aishizu@med.hokudai.ac.jp

Abbreviations: BDNF, brain-derived neurotrophic factor; BSA, bovine serum albumin; ELISA, enzyme-linked immunosorbent assay; HUVEC, human umbilical vein endothelial cells; RT-PCR, reverse transcriptase-polymerase chain reaction; TrkB, tropomyosin receptor kinase B; VEGF, vascular endothelial growth factor. 


\section{Abstract}

Brain-derived neurotrophic factor (BDNF) is a well-known humoral protein that induces growth of neurons. Recent studies have suggested that BDNF could act as an angiogenesis inducer similar to vascular endothelial growth factor (VEGF). Angiogenin is a strong mediator of angiogenesis. It has particular characteristics both as a secreted protein and a transcription factor. After being incorporated into the cytoplasm, angiogenin is immediately transferred to the nucleus and then mediates the angiogenic effects of angiogenesis inducers, including VEGF. The aim of this study is to determine the association between BDNF and angiogenin. At first, we determined the secretion of angiogenin from human umbilical vein endothelial cells (HUVEC) induced by BDNF with enzyme-linked immunosorbent assay. Next, we determined BDNF-induced nuclear translocation of angiogenin by immunofluorescent staining. In addition, we examined the mRNA expression of angiogenin in HUVEC before and after BDNF stimulation by quantitative reverse transcriptase-polymerase chain reaction. As a result, we noted that BDNF induced angiogenin secretion and nuclear translocation without an increase in the mRNA expression in HUVEC. Furthermore, we demonstrated that BDNF-induced HUVEC proliferation was significantly suppressed when neomycin, a specific inhibitor of nuclear translocation of angiogenin, was administered. These findings indicate that nuclear translocation of angiogenin is critically involved in BDNF-induced proliferation of HUVEC. In conclusion, angiogenin contributes to angiogenesis induced by BDNF.

Key words: BDNF, VEGF, angiogenin, angiogenesis 


\section{Introduction}

Angiogenesis is a physiological phenomenon in which new vascular branches arise from the existing blood vessels [1]. Individually and by interacting with each other, vascular endothelial cells repeatedly conduct the following actions, namely, budding, elongation, and lumen and bridge formation, resulting in the generation of an organized blood vessel network in three dimensions [2]. The cell dynamics in angiogenesis are regulated comprehensively. Though essential for some physiological conditions, such as embryonic development, wound healing, and tissue regeneration, angiogenesis is also involved critically in diverse pathological conditions, including growth and invasion of carcinomas, ischemic heart diseases, and retinal degenerative diseases.

Vascular endothelial growth factor (VEGF), which is a representative angiogenesis inducer, promotes migration and proliferation of vascular endothelial cells [3]. VEGF receptor is expressed specifically on vascular endothelial cells and its expression correlates with tumor progression and poor prognosis in patients with cancer, such as gastric cancer [4-6] and lung cancer [7-9]. Correspondingly, anti-VEGF antibody is used as a therapeutic agent against such cancers.

Brain-derived neurotrophic factor (BDNF) is a humoral protein that binds to a receptor called tropomyosin receptor kinase B (TrkB) on nerve cells and then induces growth of neurons [10]. It has been demonstrated that the BDNF signal through TrkB also induced growth of cells other than nerve cells; e.g., vascular endothelial cells [11-13]. Recent studies have suggested that BDNF could act as an angiogenesis inducer similar to VEGF $[14,15]$. Although some reports suggest the activation of VEGF by BDNF [16-18], the precise mechanism of angiogenicity remains unrevealed.

Angiogenin, a strong mediator of angiogenesis, was isolated from the culture supernatant of the HT-29 human colon cancer cell line [16]. It has particular 
characteristics both as a secreted protein and a transcription factor. After being incorporated into the cytoplasm, angiogenin is immediately transferred to the nucleus and then mediates the angiogenic effects of angiogenesis inducers, including VEGF [19, 20]. Correspondingly, inhibition of the nuclear translocation of angiogenin has been shown to suppress the angiogenesis.

Several factors are notably involved in the angiogenesis. Although it becomes clearer that angiogenin plays a role in the downstream of the angiogenesis inducers, there is no report that focuses on the association between BDNF and angiogenin. In the present study, we attempted to determine the effects of BDNF on the dynamics of angiogenin. 


\section{Materials and methods}

\subsection{Cell culture}

Human umbilical vein endothelial cells (HUVEC) purchased from Lonza (Basel, Switzerland) were cultured in HuMedia-EG2 (Kurabo, Osaka, Japan) with supplement of $2 \%$ fetal bovine serum at $37^{\circ} \mathrm{C}$ in a humidified atmosphere with $5 \% \mathrm{CO}_{2}$. A fresh medium was replaced every $48 \mathrm{~h}$. Cells within passages guaranteed by the supplier were used for experiments. Prior to the experiments, cells were washed three times with serum-free EBM-2 medium (Lonza) in order to avoid the influences of putative angiogenic factors in the culture medium.

\subsection{Reagents}

Recombinant human VEGF and BDNF and neomycin were purchased from Wako Pure Chemical Industries (Osaka, Japan).

\subsection{Enzyme-linked immunosorbent assay (ELISA) to quantify angiogenin}

HUVEC were grown up to 60-70\% confluency in 24-well plates. After washing, the cells were incubated with $\operatorname{BDNF}(0,5,25,50,200,500$, and 1,000 ng/mL) dissolved in EBM-2 for $24 \mathrm{~h}$ at $37^{\circ} \mathrm{C}$. Thereafter, the culture supernatant was collected and subjected to enzyme-linked immunosorbent assay (ELISA) for quantification of angiogenin using Human Angiogenin Quantikine ELISA Kit (R\&D Systems, Minneapolis, USA) according to the manufacturer's instructions. Similar experiments were repeated more than three times. Each measurement was carried out in duplicates.

\subsection{Lactate dehydrogenase (LDH) release assay}

HUVEC (2.5 × 10\%/well of 96-well plates) were exposed to $0,15.625,31.25$, 
62.5, 125, 250, 500, or $1000 \mathrm{ng} / \mathrm{mL}$ of BDNF at $37{ }^{\circ} \mathrm{C}$. After $48 \mathrm{~h}$, lactate dehydrogenase (LDH) release from the HUVEC was determined using Cytotoxicity LDH Release Assay Kit (Dojindo, Kumamoto, Japan). Assay was carried out in triplicates. Percent cytotoxicity was determined according to the manufacturer's protocol.

\subsection{Immunofluorescent staining of angiogenin}

HUVEC were grown up to about $60 \%$ confluency in 4 -well chamber slides (Corning, Corning, USA). After washing, the cells were incubated in EBM-2 without stimulant (as negative control), with $10 \mathrm{ng} / \mathrm{mL}$ of VEGF (as positive control), or with 1 $\mu \mathrm{g} / \mathrm{mL}$ of BDNF for $4 \mathrm{~h}$ at $37^{\circ} \mathrm{C}$. Thereafter, the cells were fixed with cold methanol for 10 min. After removal of methanol, the cells were washed three times with PBS. Next, in order to block non-specific binding of antibodies, the cells were soaked in PBS containing bovine serum albumin (BSA) (30 mg/mL) for $1 \mathrm{~h}$ at room temperature. Following these preparations, immunofluorescent staining of angiogenin was carried out. For this purpose, anti-human angiogenin monoclonal antibody (MANG1, mouse IgM) (BMA Biomedicals, Augst, Switzerland) was used as a primary antibody. The primary antibody was diluted to $50 \mu \mathrm{g} / \mathrm{mL}$ in the BSA-containing PBS, and the cells were allowed to react with the diluted primary antibody for $1 \mathrm{~h}$ at room temperature (alternatively, overnight at $4{ }^{\circ} \mathrm{C}$ ). After washing three times with PBS, the cells were next allowed to react with Alexa Fluor 488-labeled goat anti-mouse IgM antibody (Abcam, Cambridge, UK) for $30 \mathrm{~min}$ at room temperature in the dark. The secondary antibody was diluted to $10 \mu \mathrm{g} / \mathrm{mL}$ in the BSA-containing PBS. After washing three times with PBS followed by removal of the chambers, the cells were mounted using VECTASHIELD Mounting Medium containing 4',6-diamidino-2-phenylindole (DAPI) (VECTOR Laboratories, Burlingame, USA). These slides were observed under a 
fluorescent microscopy. Similar experiments were repeated more than three times.

\subsection{Photomicrographs}

After verifying the general state of staining under low power fields of view, photomicrographs were taken using Nikon NIS-Elements software (Tokyo, Japan). Angiogenin granules in the nucleus were counted concerning 20 cells and then the counts per nucleus were calculated.

\subsection{Quantitative reverse transcriptase-polymerase chain reaction (RT-PCR)}

HUVEC were grown up to about $60 \%$ confluency in $6 \mathrm{~cm}$ dishes. After washing, the cells were incubated in EBM-2 without stimulant (as negative control), with $10 \mathrm{ng} / \mathrm{mL}$ of VEGF (as positive control), or with $1 \mu \mathrm{g} / \mathrm{mL}$ of BDNF for $4 \mathrm{~h}$ at 37 ${ }^{\circ} \mathrm{C}$. Alternatively, HUVEC were treated with $1 \mu \mathrm{g} / \mathrm{mL}$ of BDNF at $37^{\circ} \mathrm{C}$ for 1,4 , or $24 \mathrm{~h}$. Total RNA was extracted from the cells using RNeasy mini kit (Qiagen, Hilden, Germany) according to the manufacturer's instructions. Conversion to cDNA was achieved using GoScript ${ }^{\mathrm{TM}}$ Reverse Transcription System (Promega, Madison, USA). Quantitative reverse transcriptase-polymerase chain reaction (RT-PCR) for angiogenin was carried out using StepOne®Plus Real-Time PCR Systems (Applied Biosystems, City of Foster City, USA) with the following primers: 5'-ATGGCAACAAGCGCAGCATC-3' as a forward primer and 5'-CGGACGACGGAAAATTGACTG-3' as a reverse primer. After denaturation at $95^{\circ} \mathrm{C}$ for $10 \mathrm{~min}$, the templates were allowed to amplify with 40 cycles of reaction at $95^{\circ} \mathrm{C}$ for $15 \mathrm{~s}$ and at $63^{\circ} \mathrm{C}$ for $60 \mathrm{~s}$. The amounts of angiogenin cDNA in the samples were standardized by the housekeeping glyceraldehyde 3-phosphate dehydrogenase (GAPDH) gene (5'-CCATCACCATCTTCCAGGAG-3' as a forward primer and 5'-CCTGCTTCACCACCTTCTTG-3' as a reverse primer) in the simultaneous RT-PCR. 
Relative mRNA expression of angiogenin was determined by the comparative Ct method. Similar experiments were repeated more than three times. Each PCR was carried out in triplicates.

\subsection{BDNF-induced Proliferation of HUVEC and effects of inhibitor of angiogenin nuclear translocation}

HUVEC were grown up to about $60 \%$ confluency in $3.5 \mathrm{~cm}$ dishes. After washing, the cells were incubated in EBM-2 without stimulant (as negative control), with $1 \mu \mathrm{g} / \mathrm{mL}$ of BDNF, $100 \mu \mathrm{M}$ of neomycin, or both BDNF $(1 \mu \mathrm{g} / \mathrm{mL})$ and neomycin $(100 \mu \mathrm{M})$ at $37{ }^{\circ} \mathrm{C}$. Neomycin, an aminoglycoside antibiotic, has been shown as a specific inhibitor of nuclear translocation of angiogenin [21]. After $24 \mathrm{~h}$, the cell number was counted. Simultaneously, immunofluorescent staining for angiogenin was carried out as aforementioned. Similar experiments were repeated three times.

\subsection{Statistical analysis}

Data were compared using Student's t-test according to the difference in variance determined by the F-test. The p-values of less than 0.05 were regarded as significant. 


\section{Results}

\subsection{Secretion of angiogenin from HUVEC by BDNF stimulation}

Secretion of angiogenin from HUVEC by BDNF stimulation for $24 \mathrm{~h}$ was determined using the ELISA kit. The concentration of angiogenin in the culture supernatants was increased by BDNF dose-dependently and was significantly higher in the supernatants of HUVEC stimulated by $1 \mu \mathrm{g} / \mathrm{mL}$ of BDNF than in the HUVEC without stimulation (Figure 1a). No significant release of LDH from the HUVEC treated with BDNF (even at the concentration of $1 \mu \mathrm{g} / \mathrm{mL}$ for $48 \mathrm{~h}$ ) was evident (Figure 1b). The presence of angiogenin in the supernatant as a result of BDNF cytotoxicity to HUVEC is ruled out. These findings indicate that BDNF can induce secretion of angiogenin from HUVEC.

\subsection{Nuclear translocation of angiogenin by BDNF stimulation}

In order to determine that BDNF could induce translocation of angiogenin into the nucleus, immunofluorescent staining for angiogenin was conducted on HUVEC stimulated by $1 \mu \mathrm{g} / \mathrm{mL}$ of BDNF for $4 \mathrm{~h}$. For positive control, HUVEC stimulated by 10 ng/mL of VEGF were employed. As shown in Figure 2a, fluorescent granules that represented angiogenin were observed in the nuclei of HUVEC stimulated by VEGF. This finding is consistent with the previous report that demonstrates the nuclear translocation of angiogenin induced by VEGF [19]. Similarly, fluorescent granules that represented angiogenin were observed in the nuclei of HUVEC stimulated by BDNF though the cytoplasmic staining for angiogenin was much fainter compared with HUVEC stimulated by VEGF. The numbers of angiogenin granules were 22.05/nucleus of the HUVEC stimulated by BDNF and 15.94/nucleus of the HUVEC stimulated by 
VEGF, and they were significantly greater than in the HUVEC without stimulation (9.29/nucleus) (Figure 2b).

On the other hand, the mRNA expression level of angiogenin was not significantly altered in HUVEC by stimulation with VEGF (10 ng/mL) or BDNF (1 $\mu \mathrm{g} / \mathrm{mL}$ ) for $4 \mathrm{~h}$ (Figure 3a). In addition, the angiogenin mRNA expression was not significantly altered up to $24 \mathrm{~h}$ after stimulation with BDNF (1 $\mu \mathrm{g} / \mathrm{mL})$ (Figure $3 \mathbf{b})$. The collective findings suggest that both VEGF and BDNF can induce nuclear translocation of angiogenin in HUVEC without a significant increase in the mRNA expression.

\subsection{Involvement of angiogenin nuclear translocation in BDNF-induced HUVEC proliferation}

In order to determine the involvement of angiogenin nuclear translocation in BDNF-induced angiogenesis, we conducted an experiment using neomycin, a specific inhibitor of nuclear translocation of angiogenin [21]. We demonstrated that BDNF could induce proliferation of HUVEC and the BDNF-induced proliferation was significantly suppressed when the nuclear translocation of angiogenin was inhibited by neomycin (Figure 4). These findings indicate that nuclear translocation of angiogenin is critically involved in BDNF-induced proliferation of HUVEC. 


\section{Discussion}

In the present study, we have demonstrated that BDNF could induce angiogenin secretion and nuclear translocation without a significant increase in the mRNA expression and that nuclear translocation of angiogenin is critically involved in BDNF-induced angiogenesis. Kishimoto et al. reported the nuclear translocation of angiogenin after stimulation of HUVEC by angiogenesis inducers, such as VEGF, fibroblast growth factor (FGF), and epidermal growth factor (EGF) [19]. They also claimed that inhibition of the nuclear translocation of angiogenin resulted in suppression of the angiogenesis. Regarding the potential to induce nuclear translocation of angiogenin, the strong mediator of angiogenesis, BDNF has property equivalent to other angiogenesis inducers.

The mechanisms of nuclear translocation of angiogenin are discussed as follows. Moroianu and Riordan have demonstrated that the peptide sequence RRRGL (arginine-arginine-arginine-glycine-leucine), which corresponds to the residues 31 to 35 of angiogenin, mediated the nuclear translocation [22]. They have suggested that the arginine R33 could be essential for the nuclear translocation and that the arginines R31 and R32 might regulate this process. On the other hand, Li et al. have demonstrated that exogenous angiogenin translocated into the nucleus immediately after incorporation into the cytoplasm [23]. They have also claimed that the nuclear translocation of angiogenin could occur via an independent mechanism on the microtubules and lysosomes without requirement of tyrosine kinase activation because the dynamics was not affected by either destruction of the microtubule system or inhibition of lysosomes and tyrosine kinases. On the contrary, Hu et al. have demonstrated that neomycin, an aminoglycoside antibiotic, inhibits nuclear translocation of angiogenin [21]. Based on the finding that another phospholipase C inhibitor, U-73122, has a similar effect, they suggest a pivotal 
role of the phospholipase C-inhibiting activity of neomycin on the inhibition of nuclear translocation of angiogenin. Further studies are needed to clarify the mechanisms of nuclear translocation of angiogenin induced by BDNF.

The mRNA expression analysis in HUVEC after BDNF stimulation has revealed that BDNF did not augment the angiogenin mRNA expression in HUVEC similar to VEGF. Although the angiogenin gene locates in the middle of the RNase A superfamily gene cluster, the ribonuclease activity is reported to be very low [24]. In addition, we have observed the secretion of angiogenin from HUVEC stimulated by BDNF. The collective findings suggest that BDNF effects on the protein dynamics of angiogenin rather than the transcriptional regulation. These findings appear to be consistent with the faint immunofluorescent staining of angiogenin in the cytoplasm of HUVEC after BDNF stimulation. In contrast, Arakawa et al. have demonstrated that stimulation of VEGF did not induce angiogenin secretion from co-cultured HUVEC and human skin fibroblasts [25]. Therefore, BDNF is considered to lead the nuclear translocation of angiogenin via a pathway different from VEGF.

In summary, this study clearly indicates the contribution of angiogenin to angiogenesis induced by BDNF. 


\section{Funding}

None.

\section{Author Contributions}

Designed study: AM, NH, TM, and AI. Performed the experiments: AM and YN. Analyzed the data: AM, YN, MY, YN, SM, UT, and AI. Wrote the manuscript: AM, UT, and AI.

\section{Conflict of interest statement}

The authors declare no competing financial interest. 


\section{References}

1 F. Hillen, A. W. Griffioen, Tumour vascularization: sprouting angiogenesis and beyond, Cancer Metastasis Rev. 26 (3-4) (2007) 489-502, doi: 10.1007/s10555-007-9094-7

2 K. Sugihara, K. Nishiyama, S. Fukuhara, et al., Autonomy and non-autonomy of angiogenic cell movements revealed by experiment-driven mathematical modeling, Cell Rep. 13 (9) (2015) 1814-1827, doi: 10.1016/j.celrep.2015.10.051

3 K. S. Siveen, K. Prabhu, R. Krishnankutty, et al., Vascular endothelial growth factor (VEGF) signaling in tumour vascularization: potential and challenges, Curr. Vasc. Pharmacol. 15 (4) (2017) 339-351, doi: 10.2174/1570161115666170105124038

4 K. Mizokami, Y. Kakeji, S. Oda, et al., Clinicopathologic significance of hypoxia-inducible factor $1 \alpha$ overexpression in gastric carcinomas, J. Surg. Oncol. 94 (2) (2006) 149-154, doi: 10.1002/jso.20568

5 O. Vidal, A. Soriano-Izquierdo, M. Pera, et al., Positive VEGF immunostaining independently predicts poor prognosis in curatively resected gastric cancer patients: results of a study assessing a panel of angiogenic markers, J. Gastrointest. Surg. 12 (6) (2008) 1005-1014, doi: 10.1007/s11605-007-0336-3

6 J. Chen, D. Tang, S. Wang, et al., High expressions of galectin-1 and VEGF are associated with poor prognosis in gastric cancer patients, Tumour Biol. 35 (3) (2014) 2513-2519, doi: 10.1007/s13277-013-1332-8

7 G. Fontanini, S. Vignati, L. Boldrini, et al., Vascular endothelial growth factor is associated with neovascularization and influences progression of non-small cell lung carcinoma, Clin. Cancer Res. 3 (6) (1997) 861-865

8 R. M. Bremnes, C. Camps, R. Sirera, Angiogenesis in non-small cell lung cancer: the prognostic impact of neoangiogenesis and the cytokines VEGF and bFGF in 
tumours and blood, Lung Cancer 51 (2) (2006) 143-158, doi: 10.1016/j.lungcan.2005.09.005

9 X. L. Liu, L. D. Liu, S. G. Zhang, et al., Correlation between expression and significance of delta-catenin, CD31, and VEGF of non-small cell lung cancer, Genet. Mol. Res. 14 (4) (2015) 13496-13503, doi: 10.4238/2015.October.28.10

10 S. J. Allen, D. Dawbarn, Clinical relevance of the neurotrophins and their receptors, Clin. Sci. (Lond.) 110 (2) (2006) 175-191, doi: 10.1042/CS20050161

11 M. J. Donovan, M. I. Lin, P. Wiegn, et al., Brain derived neurotrophic factor is an endothelial cell survival factor required for intramyocardial vessel stabilization, Development 127 (21) (2000) 4531-4540

12 P. Kermani, D. Rafii, D. K. Jin, et al., Neurotrophins promote revascularization by local recruitment of $\operatorname{TrkB}^{+}$endothelial cells and systemic mobilization of hematopoietic progenitors, J. Clin. Invest. 115 (3) (2005) 653-663, doi: 10.1172/JCI22655

13 S. Matsuda, T. Fujita, M. Kajiya, et al., Brain-derived neurotrophic factor induces migration of endothelial cells through a TrkB-ERK-integrin $\alpha \mathrm{V} \beta 3$-FAK cascade, J. Cell Physiol. 227 (5) (2012) 2123-2129, doi: 10.1002/jcp.22942

14 S. M. Huang, C. Lin, H. Y. Lin, et al., Brain-derived neurotrophic factor regulates cell motility in human colon cancer, Endocr. Relat. Cancer 22 (3) (2015) 455-464, doi: 10.1530/ERC-15-0007

15 A. Y. Fouda, A. Alhusban, T. Ishrat, et al., Brain-derived neurotrophic factor knockdown blocks the angiogenic and protective effects of angiotensin modulation after experimental stroke, Mol. Neurobiol. 54 (1) (2017) 661-670, doi; $10.1007 / \mathrm{s} 12035-015-9675-3$

16 H. Kim, Q. Li, B. L. Hempstead, et al., Paracrine and autocrine functions of brain-derived neurotrophic factor (BDNF) and nerve growth factor (NGF) in 
brain-derived endothelial cells, J. Biol. Chem. 279 (32) (2004) 33538-33546, doi: 10.1074/jbc.M404115200

17 C. Y. Lin, S. Y. Hung, H. T. Chen, et al., Brain-derived neurotrophic factor increases vascular endothelial growth factor expression and enhances angiogenesis in human chondrosarcoma cells, Biochem. Pharmacol. 91 (4) (2014) 522-533, doi: 10.1016/j.bcp.2014.08.008

18 Z. Zhang, Y. Zhang, Z. Zhou, et al., BDNF regulates the expression and secretion of VEGF from osteoblasts via the TrkB/ERK1/2 signaling pathway during fracture healing, Mol. Med. Rep. 15 (3) (2017) 1362-1367, doi: 10.3892/mmr.2017.6110

19 K. Kishimoto, S. Liu, T. Tsuji, et al., Endogenous angiogenin in endothelial cells is a general requirement for cell proliferation and angiogenesis, Oncogene 24 (3) (2005) 445-456, doi: 10.1038/sj.onc.1208223

20 S. Hirukawa, K. A. Olson, T. Tsuji, et al., Neamine inhibits xenografic human tumor growth and angiogenesis in athymic mice, Clin. Cancer Res. 11 (24 Pt 1) (2005) 8745-8752, doi: 10.1158/1078-0432.CCR-05-1495

21 G. F. Hu, Neomycin inhibits angiogenin-induced angiogenesis, Proc. Natl. Acad. Sci. USA. 95 (8) (1998) 9791-9795

22 J. Moroianu, J. F. Riordan JF, Identification of the nucleolar targeting signal of human angiogenin, Biochem. Biophys. Res. Commun. 203 (3) (1994) 1765-1772, doi: 10.1006/bbrc.1994.2391

23 R. Li, J. F. Riordan, G. Hu, Nuclear translocation of human angiogenin in cultured human umbilical artery endothelial cells is microtubule and lysosome independent, Biochem. Biophys. Res. Commun. 238 (2) (1997) 305-312

24 J. Sheng, Z. Xu, Three decades of research on angiogenin: a review and perspective, Acta Biochem. Biophys. Sin. 48 (5) (2016) 399-410, doi: 
10.1093/abbs/gmv131

25 M. Arakawa, T. Someno, M. Kawada M, et al., A new terrein glucoside, a novel inhibitor of angiogenin secretion in tumor angiogenesis, J. Antibiot. (Tokyo) 61 (7) (2008) 442-448, doi: 10.1038/ja.2008.60 

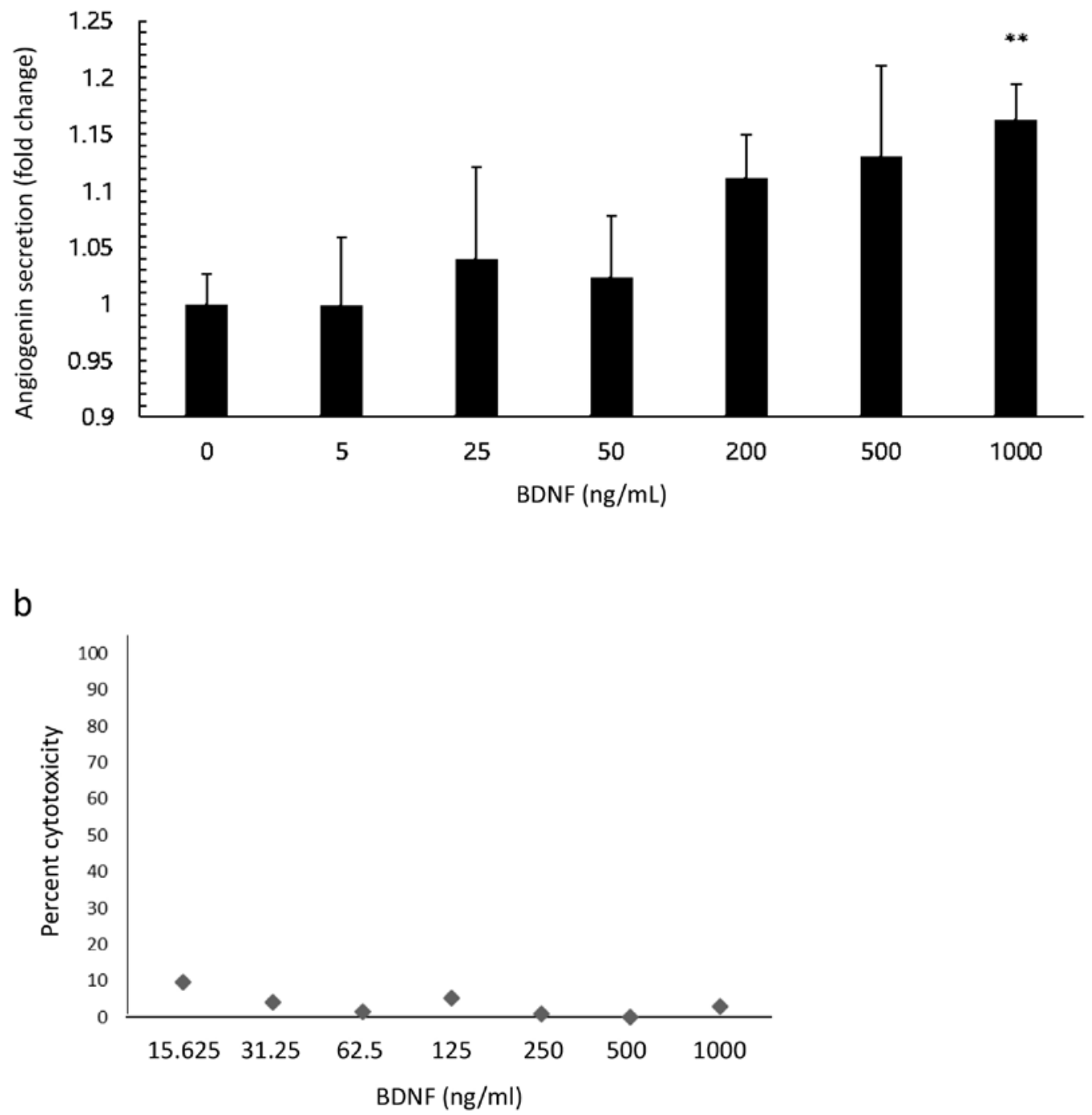

Fig. 1. Secretion of angiogenin from HUVEC by BDNF stimulation

(a) Secretion of angiogenin from HUVEC by BDNF stimulation for $24 \mathrm{~h}$ determined using ELISA kit is represented as fold change to the concentration of angiogenin in the supernatants of HUVEC without BDNF stimulation. ${ }^{* *} \mathrm{p}<0.01$ to $0 \mathrm{ng} / \mathrm{mL}$ of BDNF. (b) LDH release from HUVEC exposed to BDNF for $48 \mathrm{~h}$ determined using Cytotoxicity LDH Release Assay Kit. Data are presented as percent cytotoxicity. 
a

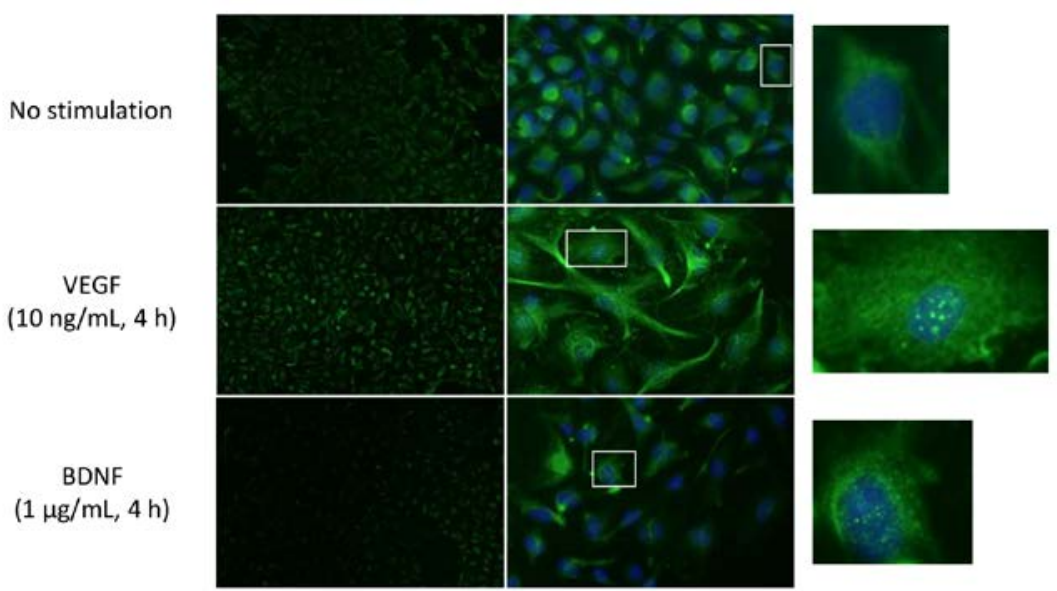

b

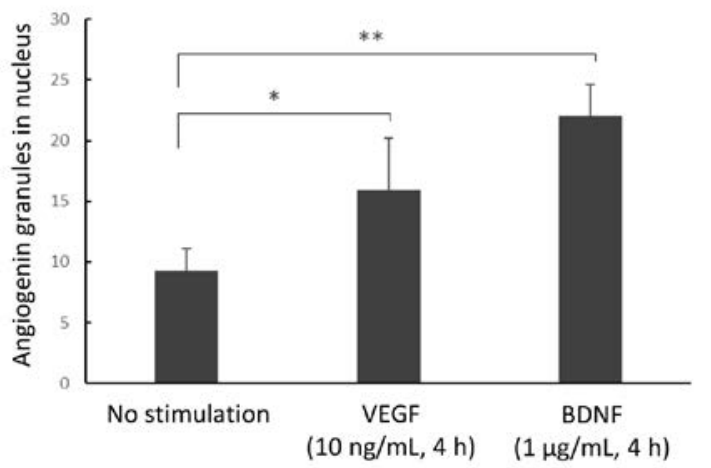

Fig. 2. Nuclear translocation of angiogenin by BDNF stimulation

(a) Immunofluorescent staining for angiogenin of HUVEC without stimulation, with VEGF stimulation, and with BDNF stimulation. Angiogenin: green, nucleus: blue. The left and middle panels represent the low and high power fields of view, respectively. The right panels represent the close-up scenes of the insets in the middle panels. (b) Number of angiogenin granules per nucleus. ${ }^{*} \mathrm{p}<0.05$, ${ }^{* *} \mathrm{p}<0.01$. 

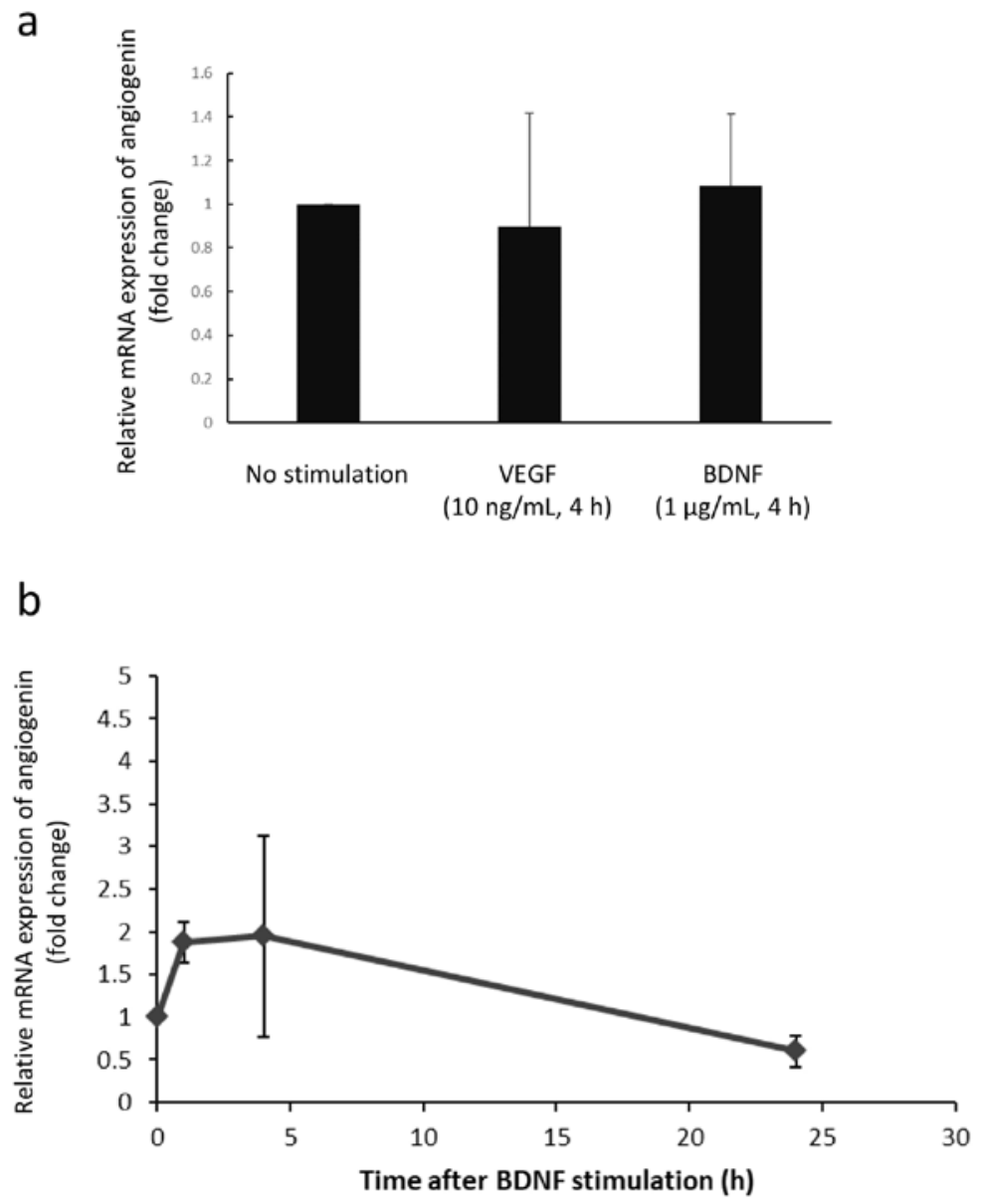

Fig. 3. Angiogenin mRNA expression in HUVEC

(a) The relative mRNA expression of angiogenin in HUVEC treated with VEGF (10 $\mathrm{ng} / \mathrm{mL}$ ) and BDNF (1 $\mu \mathrm{g} / \mathrm{ml})$ for $4 \mathrm{~h}$ was determined using real-time RT-PCR. (b) Time-dependent mRNA expression of angiogenin in HUVEC treated with $1 \mu \mathrm{g} / \mathrm{ml}$ of BDNF. The expression in HUVEC without stimulation was set as 1. Data are presented as mean \pm standard error. 


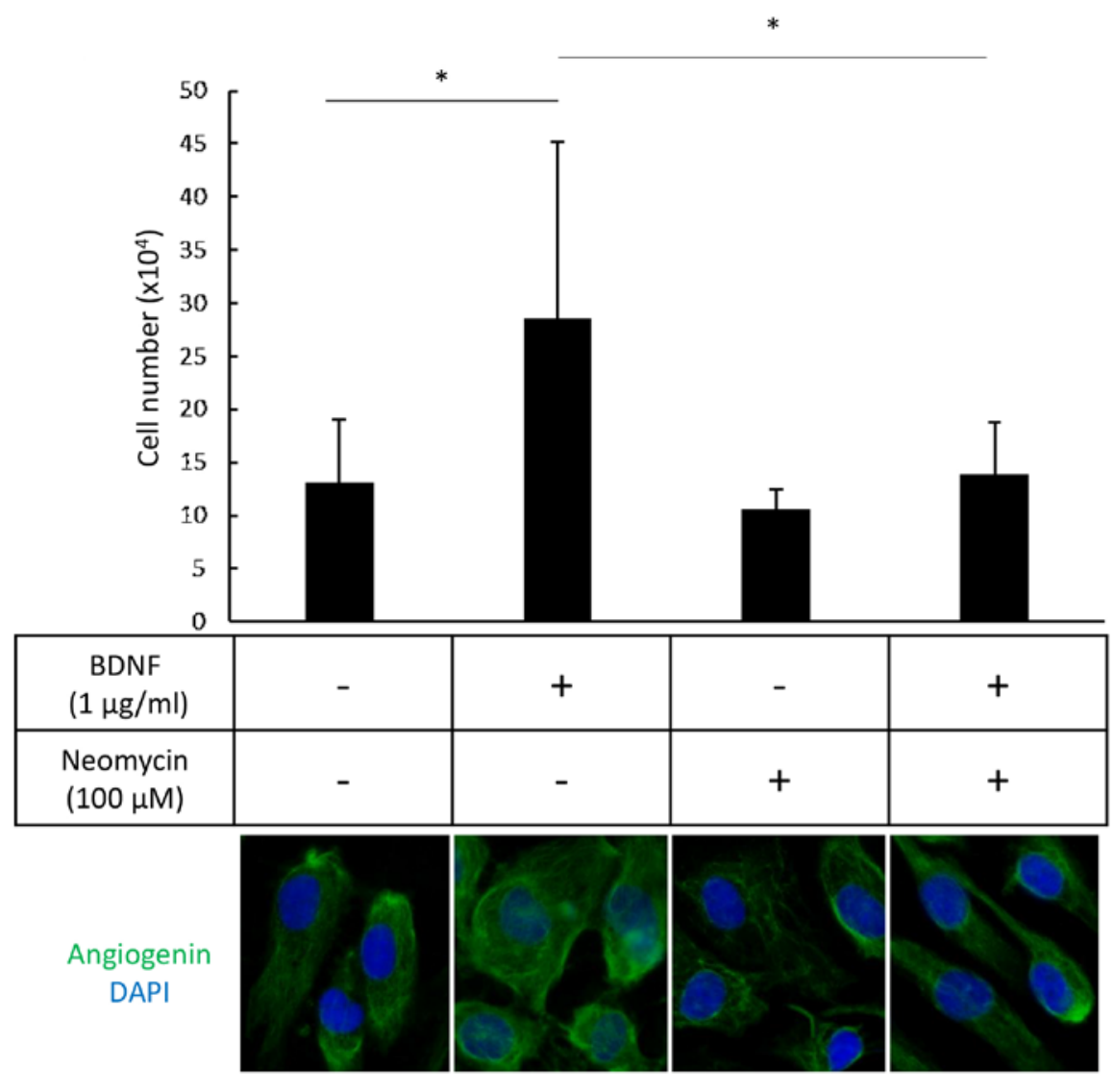

Fig. 4. BDNF-induced proliferation of HUVEC and effects of inhibitor of angiogenin nuclear translocation

HUVEC were treated with $1 \mu \mathrm{g} / \mathrm{mL}$ of BDNF, $100 \mu \mathrm{M}$ of neomycin, or both BDNF (1 $\mu \mathrm{g} / \mathrm{mL})$ and neomycin $(100 \mu \mathrm{M})$ at $37^{\circ} \mathrm{C}$. After $24 \mathrm{~h}$, the cell number was counted. Simultaneously, immunofluorescent staining for angiogenin was carried out. 